allow data sharing between hospitals and facilitates laboratory workflow, patient management and enhances public health research.

\section{IDDF2020-ABS-0153 LATISSIMUS DORSI FLAP TO REPAIR A RECURRENT EVENTRATION OF THE DIAPHRAGM: A NOVEL TECHNIQUE}

${ }^{1}$ Veerabhadra Radhakrishna*, ${ }^{2}$ Chittur Narendra Radhakrishnan. ${ }^{1}$ Bangalore Medical College and Research Institute, India; ${ }^{2}$ Manipal Hospitals, India

\subsection{6/gutjin-2020-IDDF.98}

Background Eventration of the diaphragm is a rare congenital anomaly characterized by an abnormal elevation of a part or

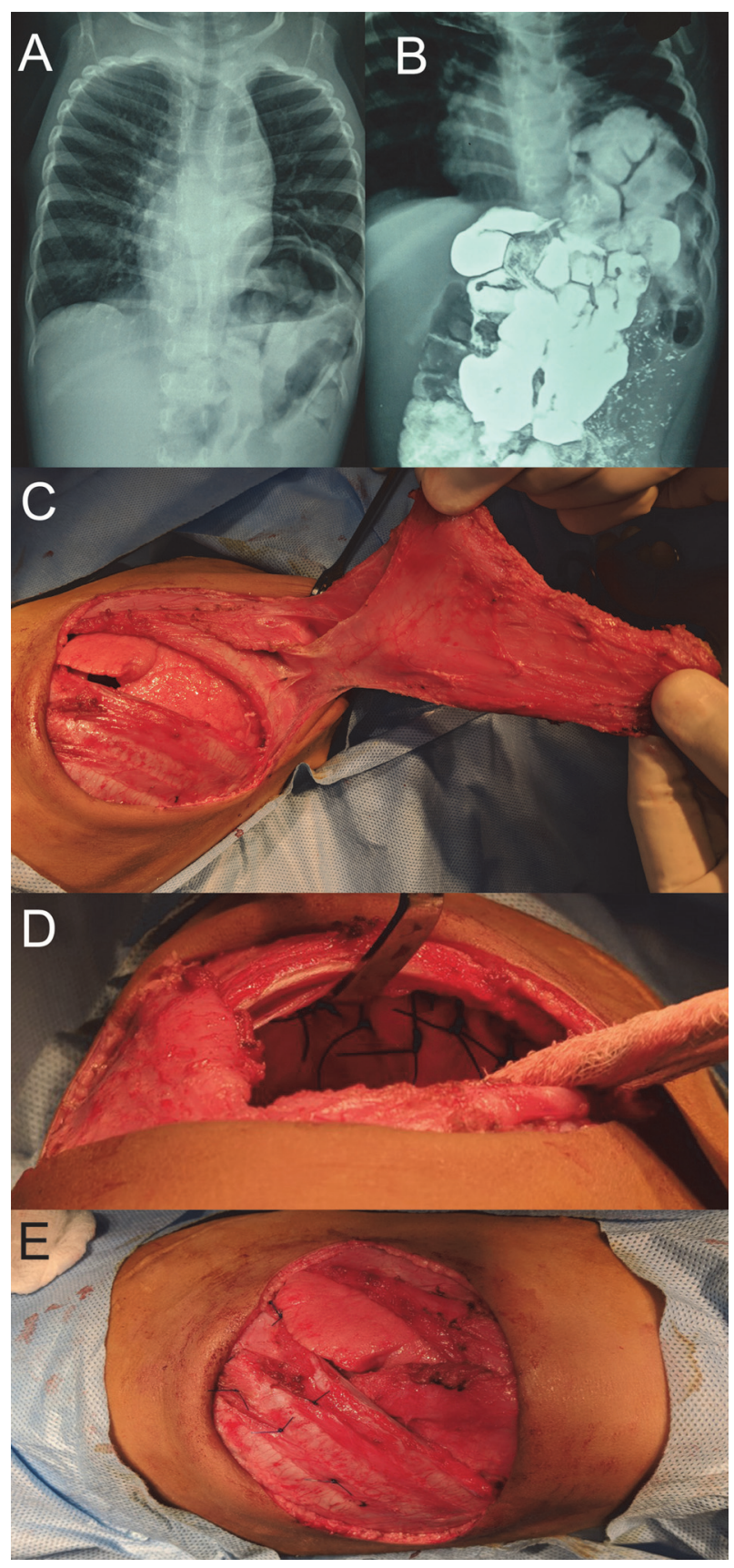

Abstract IDDF2020-ABS-0153 Figure 1 whole of the hemidiaphragm. Surgical repair is indicated in symptomatic patients and the care should always be taken to overcorrect to prevent a recurrence. As the eventrated part of the diaphragm is diffusely replaced by fibroelastic tissue, there is a high tendency to recur even after the repair of eventration. The literature on the recurrence of eventration and its repair is scarce. Here, we report a case of recurrent eventration of the diaphragm in an infant managed by latissimus dorsi flap.

Methods An 11-month-old-female infant with a past history of surgery for left-sided eventration of the diaphragm presented with recurrent lower respiratory tract infections.

On examination, she was active, alert, and pink. Her heart rate was 110 beats a minute, respiratory rate was 52 breaths per minute, and the oxygen saturation was $91 \%$ in room air. Air entry was reduced over the left lung areas. An abdominal examination was normal, except for a left subcostal scar.

Plain X-ray chest showed a recurrence of eventration (figure 1A). An upper gastrointestinal contrast study revealed the stomach and splenic flexure occupying the eventration and no evidence of gastro-oesophageal reflux (figure 1B). She was planned for plication repair with latissimus dorsi flap reinforcement.

Results Intraoperatively, a left-sided latissimus dorsi flap was identified, dissected, and safeguarded (figure 1C). Thoracotomy was done in the 5 th intercostal space. The eventrated diaphragm was plicated (figure 1D) and reinforced with left latissimus dorsi flap (figure 1E).

The follow-up was uneventful. She was thriving well at two years follow-up. To the best of our knowledge, this is the first case where latissimus dorsi flap has been used as dynamic recruitment between the thoracic and the abdominal cavity for managing recurrent diaphragmatic eventration.

Conclusions The latissimus dorsi flap reinforcement is a novel and reliable technique to treat recurrent eventration of the diaphragm. The inherent motor tone of latissimus dorsi resists the intraabdominal pressure and prevents the recurrence of diaphragmatic eventration.

\section{IDDF2020-ABS-0154 FACTORS ASSOCIATED WITH FAILED NON-OPERATIVE REDUCTION OF INTUSSUSCEPTION IN CHILDREN}

Veerabhadra Radhakrishna, Bahubali Deepak Gadgade. Bangalore Medical College and Research Institute, India

\subsection{6/gutjnl-2020-IDDF.99}

Background The non-operative method of reduction is the gold standard treatment for intussusception in children. The successful non-operative reduction of intussusception carries less morbidity and mortality, but the literature on the risk factors for failed intussusception reduction is scarce. Hence a study was conducted to evaluate the factors associated with the failure of non-operative reduction of intussusception in children.

Methods A retrospective study was conducted in a Paediatric Surgery hospital, which included children admitted with intussusception between November 2013 and February 2020.

Results A total of 106 (67\%) children underwent pneumatic reduction. Eighty-nine (84\%) children had a successful 


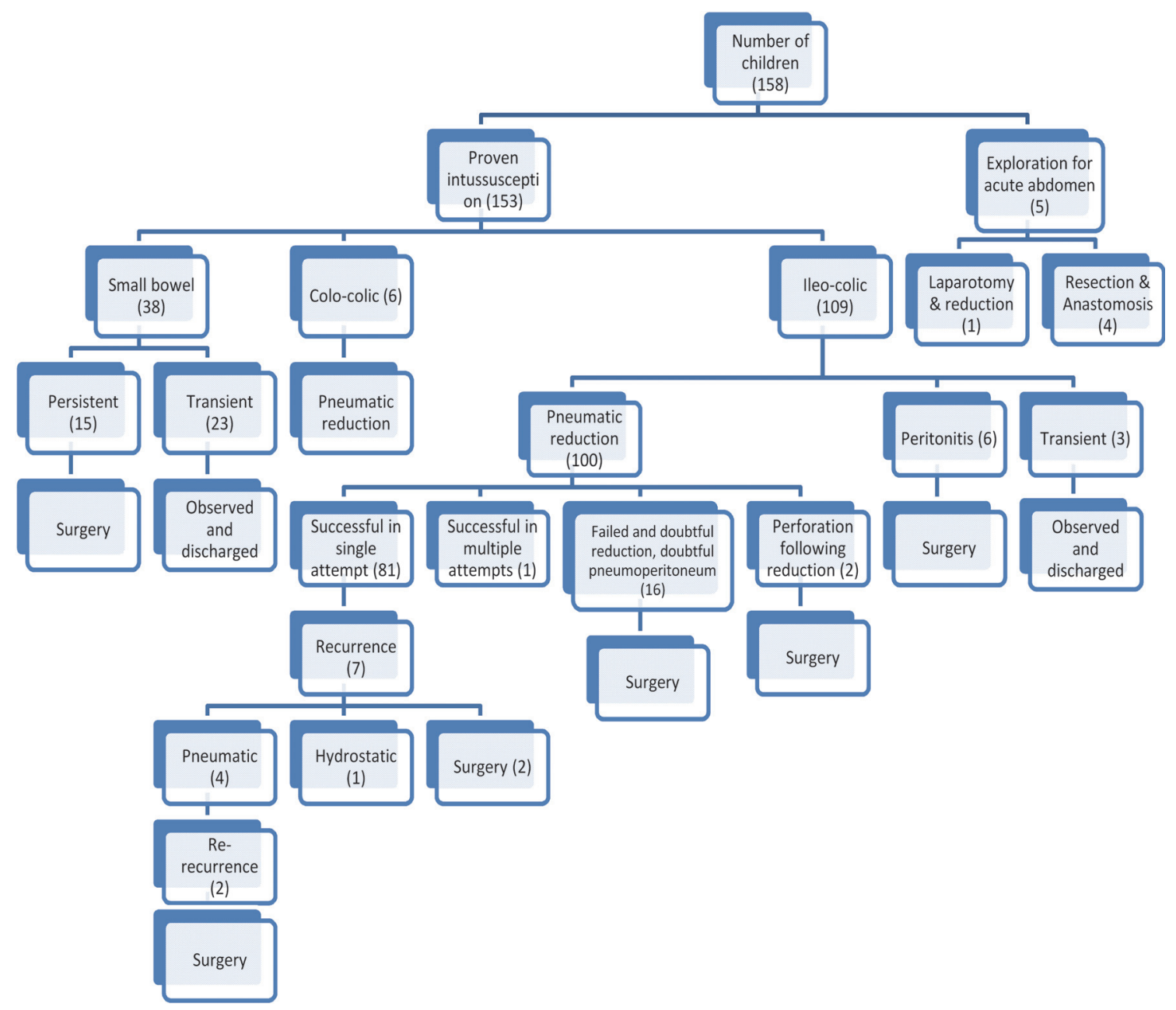

Abstract IDDF2020-ABS-0154 Figure 1 Presentation and management of intussusception

reduction. Fourteen (13\%) children with failed reduction underwent laparotomy. Twelve of these fourteen children had to undergo bowel resection (seven gangrene and five lead points) and the rest two had a successful operative reduction. Two (2\%) children with perforation following reduction, a child (1\%) each with doubtful reduction and doubtful pneumoperitoneum underwent surgery. Seven (8\%) of 89 successful reductions had a recurrence. The management of these children is depicted in figure 1.

A higher rate of failed reduction was found in children who presented at or after 48 hours of the onset of symptoms $(\mathrm{p}-0.03)$ and abdominal distension at presentation $(\mathrm{p}<0.002)$. On multiple logistic regression analysis, the children presenting at or after 48 hours onset of symptoms (OR: 11.3/p-0.039) and abdominal distension at presentation (OR: 4.46/p-0.021) were found to be associated with increased risk of failure of non-operative reduction. The variables age $<1$ year (OR:0.466/p-0.165), weight <10 kg (OR: 1.641/p-0.468), pain abdomen (OR: 1/p-0.99), vomiting (OR: 0.562/p-0.39), bilious vomiting (OR: 6.75/p-0.136), fever (OR: 2.23/p-0.357), bleeding per rectum (OR: $2.55 / \mathrm{p}-0.162)$, and palpable mass (OR: $2.74 / \mathrm{p}-0.135)$ were not associated with the failed non-operative reduction.

Conclusions The risk factors for failed non-operative reduction of intussusception include a presentation at or after 48 hours of the onset of symptoms and the presence of abdominal distension at presentation.

\section{IDDF2020-ABS-0155 A NEW DIAGNOSTIC INDEX OF SARCOPENIA FOR PREDICTING SHORT- TERM POSTOPERATIVE COMPLICATIONS IN PATIENTS UNDERGOING SURGERY FOR GASTRIC CANCER}

Xiaolong Ge*, Wei Zhou. Sir Run Run Shaw Hospital, School of Medicine, Zhejiang University, China

\subsection{6/gutjnl-2020-IDDF.100}

Background Patients with gastric cancer usually have weight loss, sarcopenia and malnutrition. Serum creatinine/cystatin C (CCR) is a new, simple, easily measured tool that could serve as a biomarker of sarcopenia. The purpose of our study was to assess the predictive ability of preoperative CCR for shortterm prognosis in patients with gastric cancer undergoing surgery.

Methods This retrospective study included 309 patients with gastric cancer undergoing surgery. Univariate and multivariate analyses were performed to identify risk factors. Patients were divided into two groups according to the optimal cut-off value of CCR. The clinical association of CCR with characteristics and postoperative complications was evaluated.

Results Among all the patients, age, lymphocyte count and cystatin C (CysC) level were independent risk factors, while red blood cell (RBC) count and CCR were independent protective factors for short-term postoperative 